\title{
INTROGRESSION IN LABORATORY POPULATIONS OF DROSOPHILA PERSIMILIS AND D. PSEUDOOBSCURA
}

\author{
LEIGH VAN VALEN \\ Zoology Deportment, University College, London
}

\section{INTRODUCTION}

Received $24 \cdot x \cdot 62$

Drosophila persimilis and $D$. pseudoobscura are sibling species almost completely isolated reproductively from each other (see the discussion by Dobzhansky, I95I). Only two hybrid individuals out of more than 50,00o individuals chromosomally examined have been found in nature (Dobzhansky, personal communication). Sexual isolation is present but incomplete (review by Dobzhansky, I95I).

The present experiment was undertaken in order to see what would happen when $D$. persimilis and $D$. pseudoobscura were put together in population cages, with a greater-than-normal advantage for hybrids. Three resuits were possible : elimination of one of the species, coexistence of both species, and the formation of a hybrid swarm by introgression. If the species had coexisted for a sufficient period it is possible that selection for reproductive isolation would have occurred, as it did in the differently designed experiment of Koopman (1950) with the same mutants of the same species. However, only the first and third alternatives were actually found.

\section{PROCEDURE}

The recessive mutants orange (or) of $D$. persimilis and glass $(g l)$ of $D$. pseudoobscura were used. Both these mutants affect the eyes. or is located near the terminal heterochromatin containing the centromere of the third chromosome and $g l$ on the distal part of the second chromosome (Tan, 1937). The hybrid between them has eyes with a visibly normal phenotype and, as will be shown below, is more fit in at least one respect than either homozygote. Nevertheless, as shown by Lancefield (1929) and others, its fertility is greatly impaired. Each of these mutant strains was outcrossed to two wild-type laboratory strains with the same gene sequence on the third chromosome, and the mutants were re-extracted.

Two hundred mutant males and 200 non-virgin mutant females of each species were placed on 3 ist July $196 \mathrm{I}$, into each of six plastic population cages of the kind described by Strickberger (1963). Three of these cages (V-15, V-i 6 and V-1 7) were kept at $\mathrm{r} 6^{\circ} \pm \mathrm{I}^{\circ} \mathrm{C}$., and three $(\mathrm{V}-\mathrm{i} 8, \mathrm{~V}-19$ and $\mathrm{V}-20)$ were kept at $25^{\circ} \pm \mathrm{I}^{\circ} \mathrm{C}$. This was done because sexual isolation between these species has been reported (Mayr and Dobzhansky, 1945) to be lower at $16 \frac{1}{2}^{\circ}$ than at $24 \frac{1}{2}^{\circ}$, and $D$. persimilis thrives better at $16^{\circ}$ than at $25^{\circ}$ (Dobzhansky, 1935). D. pseudoobscura also thrives somewhat better at the lower temperature, but its difference is not as great.

Since the development time of $D$. persimilis in bottles averages a few hours longer than that of $D$. pseudoobscura (Poulson, 1934), the period each food cup was left in the cage before replacement was varied. In $V-18$ it was 35 days; in V-19, 40 days ; in V-20, 45 days; in V-15, 52 days ; in V-16, 60 days; and in V-17, 
67 days. The generation length of $D$. pseudoobscura in comparable population cages has been estimated at about 29 days for $25^{\circ}$ and about 43 days for $15^{1^{\circ}}$ (Van Valen, L. Levine and Beardmore, I 963 ). There is no adequate published estimate of the generation length of $D$. persimilis in population cages, and there is no clear indication in the present experiment of an influence of the different lengths of food cup cycles.

Periodic egg samples and occasional counts of the entire adult populations were taken. The eggs were raised under optimal conditions at $19^{\circ}$ except where otherwise stated, and all the adults that emerged were scored for eye colour. On two occasions, most of the larvæ from supplementary bottles were dissected and their salivary chromosomes examined. Since the flies with different eye colours develop at different mean rates (see below), larvæ maturing at different times were dissected in numbers roughly proportional to those maturing in each interval.

\section{MUTANT FREQUENCIES}

As can be seen from tables $I$ and 2 , the populations at $16^{\circ}$ behaved almost identically, with the early elimination of all $D$. persimilis and

TABLE 1

Results of egg samples from the populations. In each case the left number represents phenotypically orange fies, the middle number wildtype, and the right number glass fies

\begin{tabular}{|c|c|c|c|c|c|c|}
\hline $\begin{array}{l}\text { First day } \\
\text { of sample }\end{array}$ & $V-15$ & $V-16$ & $V-17$ & $V-18$ & $V-19$ & $V-20$ \\
\hline $9^{*}$ & $14-0-392$ & $26-23-181$ & $55^{-10-105}$ & $83-5-293$ & $74-6-175$ & $120-7-186$ \\
\hline $9^{*}$ & $\ldots$ & .. & $\ldots$ & $73-9-135 \dagger$ & $124^{-1-133 \dagger}$ & . \\
\hline 25 & $\ldots$ & $\ldots$ & $\ldots$ & $0-185-591$ & $\ldots$ & $15-99-3^{89}$ \\
\hline $4^{6}$ & $2-3-192$ & $1-0-134$ & $0-0-147$ & $\ldots$ & $\cdots$ & $\cdots$ \\
\hline 53 & $\ldots$ & $\ldots$ & $\ldots$ & $\ldots$ & $0-3-9$ & $0-22-5^{6}$ \\
\hline 73 & $\ldots$ & $\ldots$ & $\ldots$ & $0-7-3^{2}$ & $0-241-117$ & $0-43-50$ \\
\hline 86 & $0-0-47$ & $0-0-59$ & $1-0-69$ & $\ldots$ & . & $\cdots$ \\
\hline 97 & .. & .. & .. & $0-18-4^{8}$ & .. & $0-11-36$ \\
\hline 114 & $\ldots$ & $\ldots$ & .. & $0-0-0$ & $0-79-33$ & $0-3^{0-5^{8}}$ \\
\hline 124 & $0-0-411$ & $0-0-3^{8} 9$ & $0-0-474$ & . & $\ldots$ & $\ldots$ \\
\hline 134 & . & ... & . & $0-2-21$ & $1-426-133$ & $0-27-31$ \\
\hline 154 & $\cdots$ & $\ldots$ & ... & $1-4-8$ & $0-117-22$ & $0-32-27$ \\
\hline 175 & $\ldots$ & $\cdots$ & $\ldots$ & $0-1-10$ & $0-188-54$ & $0-66-53$ \\
\hline 196 & $\ldots$ & $\ldots$ & ... & $\ldots$ & $0-15^{6-22}$ & $\ldots$ \\
\hline 214 & $\ldots$ & $\cdots$ & $\ldots$ & $0-0-53$ & $\cdots$ & . \\
\hline 237 & $\cdots$ & $\ldots$ & $\ldots$ & $\ldots$ & $0-376-15$ & $0-11-27$ \\
\hline $25^{6}$ & $\cdots$ & $\cdots$ & $\ldots$ & $\cdots$ & $0-47^{2-4^{8}}$ & $\ldots$ \\
\hline 275 & $\cdots$ & $\cdots$ & $\cdots$ & $\cdots$ & $0-1134-93$ & $\cdots$ \\
\hline 288 & $\cdots$ & $\cdots$ & $\cdots$ & $\cdots$ & $0-34^{2-18}$ & $\cdots$ \\
\hline
\end{tabular}

* Eggs laid by the parental flies, which were non-virgin on entering the cages.

$t$ Eggs taken from side instead of top of food.

visible hybrids. Examination of a total of 348 haploid sets of salivary chromosomes from the three cages, from eggs laid on the $124^{\text {th }}$ day, showed a single second chromosome of $D$. persimilis, all the other $\mathrm{X}$ and second chromosomes being completely $D$. pseudoobscura.

A greater or lesser degree of introgression occurred in all three populations at $25^{\circ}$. Although it can be seen from tables I and 2 that pure $D$. persimilis was eliminated rather rapidly, wild-type flies survived in each cage to its termination and made up nine-tenths or 
more of population V-I 9 in its last two or three months. Males and females were recorded separately but never gave discordant frequencies.

The occurrence of introgression at $25^{\circ}$ while $D$. persimilis was eliminated at $16^{\circ}$ was unexpected and remains unexplained.

The egg samples do not provide an entirely accurate picture of the phenotype frequencies in the population cages. The adult samples are usually not much different, but near the end of the $\mathrm{V}-\mathrm{I} 8$ population wild-type flies were much more frequent in the cage than in the egg samples. Furthermore, egg samples from the parental generation of V-r 8 and V-r 9 differed somewhat in composition according to whether they were taken from the top of the food cup, as in all subsequent samples, or from the side where part of the food has been removed,

TABLE 2

Counts of adults in the population cages. Order of phenotypes as in table I

\begin{tabular}{|c|c|c|c|c|c|c|}
\hline $\begin{array}{l}\text { Day of } \\
\text { count }\end{array}$ & V-1 5 & $V-16$ & $V-17$ & $V-18$ & $V-19$ & $V-20$ \\
\hline 2.1 & $\ldots$ & ... & $\ldots$ & $\cdots$ & $43-3^{0-1} 4^{8}$ & $\cdots$ \\
\hline 39 & $\cdots$ & ... & $\ldots$ & $7-93-186$ & $\ldots$ & $\ldots$ \\
\hline 76 & ... & . & $\ldots$ & ... & $2-3^{8} 9-124$ & $\ldots$ \\
\hline 145 & $0-0-893$ & $0-0-937$ & $0-0-725$ & . & $\ldots$ & $\ldots$ \\
\hline 180 & $\ldots$ & $\ldots$ & $\ldots$ & $0-11-126$ & $\ldots$ & $\ldots$ \\
\hline 195 & $\cdots$ & $\cdots$ & $\cdots$ & $\cdots$ & $0-470-74$ & $0-27-22$ \\
\hline 218 & $\cdots$ & $\cdots$ & ... & $0-12-96$ & .. & .. \\
\hline $26 I$ & $\cdots$ & $\ldots$ & $\cdots$ & $\ldots$ & $\cdots$ & $0-24-63$ \\
\hline $3^{10}$ & $\cdots$ & ... & $\cdots$ & $\cdots$ & $0-721-73$ & $\cdots$ \\
\hline
\end{tabular}

where most of the eggs that remain in the cage are laid. D. persimilis seemed to prefer the latter region.

The samples from the parental generation (where only the mutant frequencies are valid because of non-virginity of the introduced parents) indicate that there was some selection among the adults against $D$. persimilis in all or nearly all cages. The form of this selection is unknown.

\section{CHROMOSOME FREQUENCIES}

Since only the $\mathrm{X}$ and second chromosomes of $D$. pseudoobscura and $D$. persimilis can be distinguished cytologically (Tan, I935; Koller, 1936 ; Sturtevant and Dobzhansky, I936 ; Dobzhansky and Sturtevant, I938; Dobzhansky, I944), cytological information was available on the changes in frequency of only these chromosomes. In addition, however, the gene or gives some information about the third chromosome. Since some crossing over occurs in D. pseudoobscura despite the presence of even moderately long or complex inversions on the same chromosome (Dobzhansky and Epling, i948; R. P. Levine, I956 ; Sturtevant and Dobzhansky, I936 ; Dobzhansky and Sturtevant, I938; also see below), $\mathrm{X}$ and second chromosomes, 
cytologically of one species, may in fact contain some genes of the other. The strains of the species used differ cytologically by an inversion on each arm of the $\mathrm{X}$ chromosome and one on the rodshaped second chromosome.

On the I34th day an egg sample was taken from each $25^{\circ}$ cage for chromosome analysis. The results are shown in table 3. The numbers of $\mathrm{X}$ chromosomes are smaller than those of second chromosomes because of the presence of males, which have only a single $\mathrm{X}$ chromosome. Frequencies of each chromosomal association (e.g. pseudoobscura XL, persimilis XR, hybrid II) are not given because in no case was the association between $\mathrm{X}$ and II other than random,

TABLE 3

Results of chromosome samples of the populations

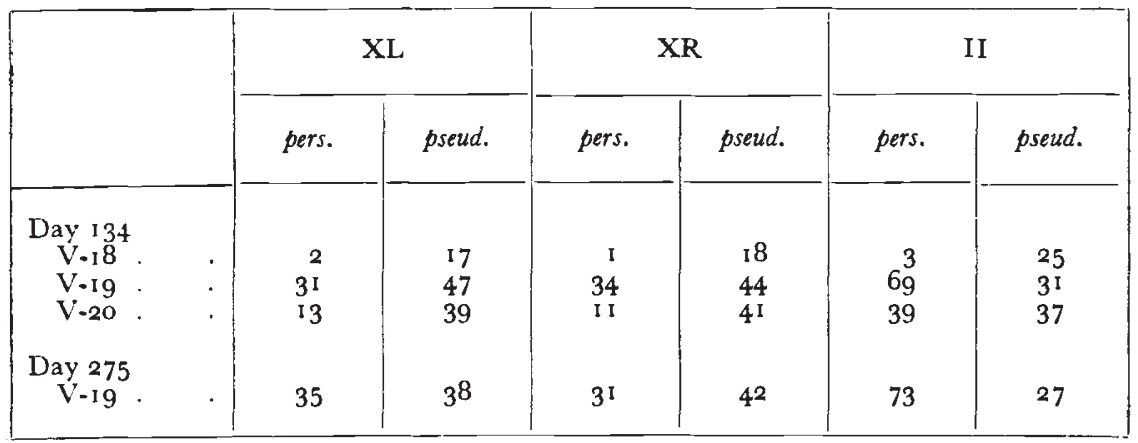

although in every case a positive association between XL and XR was possible ( $\mathrm{P}$ always less than $0 \cdot 2$, usually much less).

The frequencies for the two arms of the $\mathrm{X}$ chromosome are comparable in each population, and show a moderate to marked reduction in the $D$. persimilis gene arrangements. In populations $\mathrm{V}-\mathrm{I} 9$ and $\mathrm{V}-20$, however, there is a significantly greater proportion of $D$. persimilis second chromosomes than of $D$. persimilis $\mathrm{X}$ chromosomes.

The two estimates of the frequency of $D$. pseudoobscura second chromosomes provided by cytological analysis and examination of eye colour are in general agreement but are nevertheless significantly different for both V-ig and V-20. The latter shows that crossing over has occurred between $g l$ and the inversion and that the chromosome segments containing these elements have different adaptive values in the mixed population genome. In each case the segment with the inversion is less abundant than the segment with $\mathrm{gl}$. This is true whether the Hardy-Weinberg formula or the observed chromosome pairings (see below) are used to estimate the frequency of heterozygotes for $g l$.

In each chromosome sample (except those of V-I8, where the test is insensitive) the heterokaryotypes were significantly less frequent than expected by the Hardy-Weinberg formula, indicating the probable 
presence of an isolating mechanism, presumably in this case sexual isolation between the parents, acting before the late third larval instar. This is the reverse of the result obtained by Mettler (1957). It might seem that the intensity of this presumed sexual isolation could be crudely estimated for V-I9 and V-20 by dividing the number of heterokaryotypes by the number of homokaryotypes ( $c f$. Merrell, I 950). However, this estimate is biased because (I) the parental homokaryotypes were of unequal frequency and (2) some of the parents were presumably heterokarytotypes, but the relative fertility and mating propensities of these are unknown. The first bias can be overcome statistically but the second cannot without additional information that is not available for this generation.

Eight virgin $g l$ flies from V-Ig were put as pairs into four vials. Their offspring were raised in the usual way and nine larvæ from each pair were dissected. In at least two and at most four of the parental flies a persimilis second chromosome gene arrangement was present in heterozygous condition, thus proving the occurrence of this gene arrangement and the pseudoobscura mutant $g l$ on the same chromosome. Much the most probable cause of this is crossing over.

A second sample of chromosomes was taken on the 275th day from V-19, the only population remaining. As in the earlier sample, the association between $\mathrm{X}$ and II was random but between $\mathrm{XL}$ and $\mathrm{XR}$ it was strongly positive. The frequencics of persimilis $\mathrm{XL}$ and $\mathrm{XR}$ were not markedly different from their earlier values, suggesting the establishment of an equilibrium between 40 and 50 per cent. The cause of this apparent equilibrium is unknown, but Mettler (1957) found a similar results in his cage 6 (see also Wallace (1948) and Bennett (1958)). Despite the drop in the frequency of $g l$, the frequency of the pseudoobscura second chromosome gene arrangement was not significantly changed, suggesting an equilibrium at about 30 per cent. This is about the frequency of $g l$ if the Hardy-Weinberg formula were applicable. It does in fact appear to be applicable at this time, since the frequencies of heterokaryotypes are now not significantly different from those expected ( $\chi^{2}$ computed both independently and combined for the three classes).

\section{SEXUAL ISOLATION}

Virgin flies raised at $25^{\circ}$ from eggs and aged for about a week were used for tests of sexual isolation against the mutant strains of each pure species (raised for two generations at $25^{\circ}$ ). Ten males and five females of each of two kinds were placed in a creamer for three days at $25^{\circ}$. The females were then dissected and the presence or absence of sperm in the spermathecæ and ventral receptacles noted. Merrell (I954) has validated this method for these two species at $2 \mathrm{I}^{\circ}$.

Adults from the egg sample of V-I9 on the $275^{\text {th }}$ day were used for estimates of sexual isolation, together with flies from the parental strains hatching at the same time. The crosses and the results are 
summarised in table 4. In each creamer five homogamic females were present together with the five heterogamic females indicated. The small number of glass females from the population cage prevented further crosses.

The results are striking. There is no indication whatever of sexual isolation between the hybrid population and either parental species, despite the strong isolation between the parental species. This is reminiscent of the results of Mayr (I946), who found a generally

TABLE 4

Results of sexual isolation tests between fies from V-19 and stock flies. The first fraction of each pair represents homogamic matings, the second heterogamic matings in the same creamers. Each fraction is the number found inseminated divided by the total number dissected

\begin{tabular}{|c|c|c|c|c|}
\hline & & \multicolumn{3}{|c|}{ Heterogamic females } \\
\hline & & D. pers., or & cage, + & D. pseud., $g l$ \\
\hline $\begin{array}{l}\text { Males : } \\
\quad \text { D. pers., or }\end{array}$ & $\cdot$ & $\ldots$ & $\frac{18}{33}, \frac{15}{3^{1}}$ & $\frac{17}{31}, \frac{2}{30}$ \\
\hline cage, + . & . & $\frac{23}{30}, \frac{25}{31}$ & $\cdots$ & $\frac{20}{33}, \frac{23}{30}$ \\
\hline cage, $g l$. & . & $\frac{16}{21}, \frac{15}{23}$ & $\cdots$ & $\frac{15}{22}, \frac{13}{21}$ \\
\hline D. pseud., $g l$ & . & $\frac{24}{33}, \frac{4}{31}$ & $\frac{21}{30}, \frac{24}{32}$ & $\cdots$ \\
\hline
\end{tabular}

similar situation for $\mathrm{F}_{1}$ hybrids between the same species, and of Birch (1961), who found a similar but less extreme breakdown of isolation in a laboratory hybrid swarm of Dacus. A formal test of significance seems unnecessary here.

\section{MORPHOLOGY}

Reed, Williams and Chadwick (I942) discovered that a theoretically derived morphological index adequately separated all specimens tested of $D$. persimilis and $D$. pseudoobscura, when these specimens were raised under comparable conditions. When the index was used in combination with sex comb number (Mather and Dobzhansky, I 939) an even better separation was found. The index is the product of the wing area by the cube of the wing length.

Wild-type males from V-I9, derived by the egg sample of the $275^{\text {th }}$ day, were compared with the parental mutant strains for the wing index. All were raised at $25^{\circ}$ in optimal conditions. Wing area was taken to be the product of wing length by wing breadth, since shape aside from these components is nearly uniform. The 
results are shown in table 5 , the wing index being given in arbitrary units.

For each character the flies from V-I9 are intermediate between those of the pure species. In the wing index they are slightly closer to $D$. persimilis and in the sex comb count to $D$. pseudoobscura. The $g l$ flies from the cage are ostensibly but not significantly closer than the wild-type flies to $D$. pseudoobscura in both characters. The flies from the cage are markedly and significantly more variable than the pure species in both characters, suggesting that an integrated population genotype has not yet become completely established. An alternative possibility to explain this greater variance, that the population cage

TABLE 5

Comparison of flies from V-19 with stock flies, by a wing index and number of bristles in both sex combs. $\mathrm{s}$ represents the standard deviation of the sample

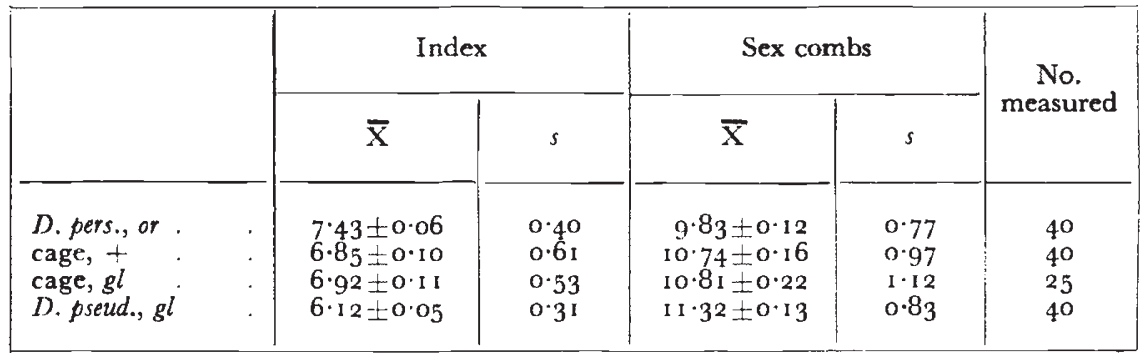

has diverse environments that flies of different wing and sex comb phenotypes exploit, has been rejected as a result of counting the teeth of sex combs on $40 \mathrm{D}$. pseudoobscura taken as eggs from a population cage (173-22) and raised under comparable conditions. The population standard deviation was 0.78 , and the mean was comparable.

\section{DEVELOPMENT TIMES}

Examination of the day-by-day counts of the flies from egg samples made it clear that those homozygous for or and $g l$ were slower in development than the wild-type ones. Table 6 summarises the data, giving the mean intra-bottle differences between the development times of the different phenotypes, using of course only those bottles in which both the classes being compared emerged. All differences are significant at the 5 per cent. level or lower. No development times were estimated for flies emerging from food cups in the cages, but the breakdown for crowded and uncrowded cultures accentuates the differences in development time.

In a population cage the selective advantage of a short development time is much greater than would be predicted by merely estimating the time an additional generation would appear for the faster genotype. This is because the first flies emerging from their 
puparia are the first to lay eggs, and their larvæ will be larger than those from eggs laid later. Later eggs may not be laid at all because of the churning mass of food and larvæ usually occupying the cup.

TABLE 6

Within-bottle comparisons of development times for three different phenotypes taken as eggs from the population cages. Each number represents the mean additional time (in days) that the phenotype on the left in each pair took in comparison to the phenotype on the right. Crowded cultures here mean those with over 200 adults of all phenotypes emerging

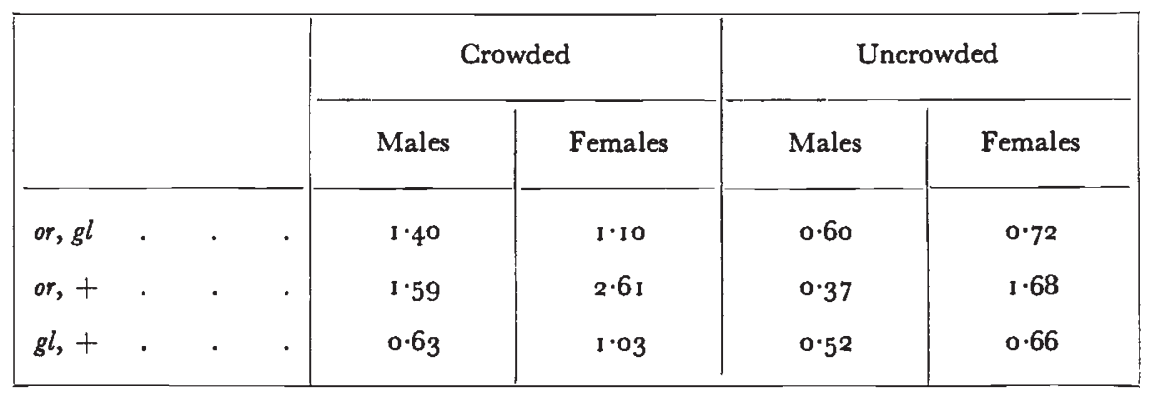

In the fiercely competitive situation of a population cage food cup it is presumed that larger larvæ have a decided advantage.

\section{DISCUSSION}

The number of individuals recorded in each sample in table I is very roughly proportional to the number of adults in the population, as judged by adult counts (table 2) and visual examination of the cages, even though the number of bottles counted is not always the same. After about the second month the adult population size of $\mathrm{V}$-r 9 became and remained noticeably larger than that of V-r 8 and $\mathrm{V}-20$.

The small size of populations V-r 8 and V-20 may reflect the action of both the advantage of development time (and perhaps others) of wild-type flies and some of the isolating mechanisms reviewed by Dobzhansky (195I). The backcross progeny are weakened and often sterile; this is reflected in the high proportion (usually more than half, in one case all) of the eggs in samples from V20 and especially $\mathrm{V}$-r 8 that failed to develop. If most of the flies that survive are hybrids, perhaps because of the situation described in the preceeding section, their progeny will be fewer than if they are of the pure species. V-r 9 also had a small adult population size for a while, but soon recovered. This recovery may have been due to a reorganisation of the genome that permitted chromosomes of both species to remain without causing undue harm to their carriers.

The differences between the three populations in the frequencies of visible and cytological chromosome markers are perhaps a result of the random occurrence of gene combinations that provided different 
receptiveness to heterospecific chromosomes in each population. It is known (Dobzhansky and Boche, 1933) that there is genetic variability within both $D$. pseudoobscura and $D$. persimilis with respect to resistance to testis degeneration in $F_{1}$ hybrids between the two species. It is plausible but unproved that similar variability exists with respect to resistance to other aspects of hybrid breakdown and sterility. The divergent courses followed by the three populations at $25^{\circ}$ may well be due to the occurrence of different gene combinations in the three populations, with a resultant divergence in selective trends and available adaptive peaks ( $c f$. Dobzhansky and Pavlovsky, 1957; Dobzhansky and Spassky, 1962 ; and Van Valen, L. Levine and Beardmore, 1963).

\section{SUMMARY}

Mutant $D$. pseudoobscura and $D$. persimilis were put into population cages at $16^{\circ} \mathrm{C}$. and $25^{\circ}$ and left undisturbed. $D$. persimilis was soon eliminated at $16^{\circ}$, but at $25^{\circ}$ a hybrid swarm was formed in each of the three populations. These hybrid swarms were analysed by use of the mutant markers, the chromosomal differences between the species, sexual isolation, and morphology. The populations were in every case intermediate between the parental species. An apparent equilibrium in the $\mathrm{X}$ as well as the second chromosome was present. The experimental flies were sexually isolated from neither parental species, and were morphologically more variable. The three populations at $25^{\circ}$ were not identical, perhaps because of different courses taken in the reorganisation of their genomes.

Acknowledgments.- The experiments described here were made while I was a Boese Postdoctoral Fellow of Columbia University. I wish to thank Phebe Van Valen, who once cared for the populations for a short time, and Th. Dobzhansky, who made laboratory facilities available.

\section{REFERENCES}

BENNETT, J. H. 1958. The existence and stability of selectively balanced polymorphism at a sex-linked locus. Australian Four. Biol. Sci., II, 598-602.

BIRCH, L. C. 196I. Natural selection between two species of tephritid fruit fly of the genus Dacus. Evolution, ${ }_{5}, 360-374$.

Dobzhansky, Th. 1935. Drosophila miranda, a new species. Genetics, 20, 377-39 1.

Dobzhansky, тh. 1944. Chromosomal races in Drosophila pseudoobscura and Drosophila persimilis. Carnegie Inst. Wash. Pub., 554, 47-144.

dobzhansky, тh. 1951. Genetics and the Origin of Species. Columbia Univ. Press, New York.

DOBZhaNsky, TH., AND BOCHE, R. D. 1933. Intersterile races of Drosophila pseudoobscura Frol. Biol. Zentralbl., 58, 589-607.

DORZHANSKY, TH., AND EPLING, c. I944. Taxonomy, geographical distribution and ecology of Drosophila pseudoobscura and its relatives. Carnegie Inst. Wash. Pub. 554, I-46.

DOBZHANSKY, TH., AND EPLiNG, C. 1948. The suppression of crossing over in inversion heterozygotes of Drosophila pseudoobscura. Proc. Nat. Acad. Sci., 34, 137-1 $4^{1}$. 
DOBZHANSKY, TH., AND PAVLOVSKY, O. 1957. An experimental study of interaction between genetic drift and natural selection. Evolution, II, 311-319.

DOBZHANSKY, TH., AND SPASSKY, N. P. 1962. Genetic drift and natural selection in experimental populations of Drosophila pseudoobscura. Proc. Nat. Acad. Sci., $48,148-156$.

DOBZHANSKY, TH., AND STURTEVANT, A. H. 1938. Inversions in the chromosomes of Drosophila pseudoobscura. Genetics, 23, 28-64.

DOBZHANSKY, Th., AND tan, c. C. 1936. Studies on hybrid sterility. III. A com. parison of the gene arrangement in two species, Drosophila pseudoobscura and D. miranda. Zeitschr. ind. Abst.-Vererb., 72, 88-1 14.

Koller, P. C. 1936. Structural hybridity in Drosophila pseudo-obscura. Four. Genet., 32, 79-102.

KOOPMAN, K. F. 1950. Natural selection for reproductive isolation between Drosophila pseudoobscura and Drosophila persimilis. Evolution, 4, 135-1 48 .

LANCEFIELD, D. E. 1929. A genetic study of crosses of two races or physiological species of Drosophila obscura. Zeitschr. ind. Abst.-Vererb., 52, 287-31 7 .

LEvine, R. P. 1956. Crossing over and inversions in coadapted systems. Amer. Nat., go, $4 \mathrm{I}-45$.

MATHER, K., AND DOBZHANSKY, TH. 1939. Morphological differences between the " races" of Drosophila pseudoobscura. Amer. Nat., 73, 5-25.

MAYR, E. 1946. Experiments on sexual isolation in Drosophila. VI. Isolation between Drosophila pseudoobscura and Drosophila persimilis and their hybrids. Proc. Nat. Acad. Sci., 32, 57-59.

MAYR, E., AND DOBZHANSKy, TH. 1945. Experiments on sexual isolation in Drosophila. IV. Modification of the degree of isolation between Drosophila pseudoobscura and Drosophila persimilis and of sexual preferences in Drosophila prosaltans. Proc. Nat. Acad. Sci., 3I, 75-82.

MERRELL, D. J. 1950. Measurement of sexual isolation and selective mating. Evolution, 4, 326-331.

MERrell, D. J. I954. Sexual isolation between Drosophila persimilis and D. pseudoobscura. Amer. Nat., 88, 93-99.

METTLER, L. E. 1957. Studies on experimental populations of Drosophila arizonensis and Drosophila mojavensis. Stud. Genet. Drosophila IX, Univ. Texas Pub. 572I, 157-181.

Poulson, D. F. 1934. Times of development of the two races of Drosophila pseudoobscura. Jour. Exper. Zool., 68, 237-245.

REED, s. C., WILliams, C. M., AND CHADWICK, L. E. 1942. Frequency of wing beat as a character for separating species, races, and geographic varieties in Drosophila. Genetics, 27, 349-361.

STRICKBERGER, M. w. 1963. Evolution of fitness in experimental population of Drosophila pseudoobscura. Evolution, $17,40-55$.

sturteVANt, A. H., AND DOBZhansky, TH. 1936. Geographic distribution and cytology of "sex ratio" in Drosophila pseudoobscura and related species. Genetics, $2 I, 473-490$.

TAN, c. C. 1935. Salivary gland chromosomes in the two races of Drosophila pseudoobscura. Genetics, 20, 392-402.

TAN, c. c. 1937. The cytological maps of the autosomes in Drosophila pseudoobscura. Zeitschr. Zellf. Mikr. Anat., 26, 439-461.

TAN, c. C. 1946. Genetics of sexual isolation between Drosophila pseudoobscura and D. persimilis. Genetics, 31, 558-573.

van VAlen, L., Levine, L., and beardmore, J. 1963. Temperature sensitivity of chromosomal polymorphism in Drosophila pseudoobscura. Genetica, 33, 113-127.

WAllace, B. 1948. Studies on "sex-ratio" in Drosophila pseudoobsoura. I. Selection and "sex-ratio". Evolution, 2, 189-217. 